\title{
Bevacizumab and ranibizumab for neovascular age-related macular degeneration: an updated meta-analysis of randomised clinical trials
}

\author{
Laurent Kodjikian • Evelyne Decullier • Eric H. Souied • \\ Jean-François Girmens • Emilie E. Durand • \\ François R. Chapuis • Laure Huot
}

Received: 18 April 2014 / Revised: 18 July 2014 / Accepted: 30 July 2014 / Published online: 22 August 2014

(C) The Author(s) 2014. This article is published with open access at Springerlink.com

\begin{abstract}
Purpose Neovascular age-related macular degeneration (AMD) is the main cause of central vision loss among individuals aged 50 years or older in developed countries. The aim of this study was to review systematically the effect of bevacizumab compared to ranibizumab in patients with $\mathrm{AMD}$ at 1 year.

Methods A systematic review was performed on Medline, Embase, and the Cochrane Library and Trial registers to
\end{abstract}

L. Kodjikian $(\bowtie)$

Service d'ophtalmologie, Hospices Civils de Lyon, Groupement Hospitalier Nord, Hôpital de la Croix-Rousse, 103 Grande Rue de la Croix-Rousse, F-69317 Lyon Cedex 04, France

e-mail: kodjikian.laurent@wanadoo.fr

L. Kodjikian · E. Decullier · F. R. Chapuis $\cdot$ L. Huot

Université de Lyon, Lyon F-69007, France

L. Kodjikian

CNRS UMR 5510 Mateis, Villeurbanne F-69621, France

E. Decullier · E. E. Durand · F. R. Chapuis · L. Huot

Pôle Information Médicale Evaluation Recherche, Unité de

recherche clinique, Hospices Civils de Lyon, Lyon F-69003, France

E. Decullier · F. R. Chapuis $\cdot$ L. Huot

Université Lyon 1, EAM 4128 Santé Individu Société,

Lyon F-69003, France

E. H. Souied

Service d'ophtalmologie, Centre Hospitalier Intercommunal de

Créteil, Créteil F-94000, France

E. H. Souied

CRC, Université Paris Est, Créteil F-94000, France

J.-F. Girmens

Centre Hospitalier d'Ophtalmologie des Quinze-Vingts,

INSERM-DHOS CIC503, Paris F-75012, France
October 2013. Eligibility criteria for selecting studies were randomised controlled trials (RCT) comparing bevacizumab with ranibizumab in patients with neovascular AMD. Odds ratio (OR) and mean difference (MD) estimates were synthesized under fixed- and random-effects models. Heterogeneity was assessed using the $\mathrm{Q}$ statistic and $\mathrm{I}^{2}$.

Results Five RCTs were included, representing 2,686 randomised patients. The meta-analysis confirmed the non-inferiority of bevacizumab compared to ranibizumab for change in visual acuity at 1 year (MD 0.57 letters, -1.80 to $\left.0.66, p=0.37, \mathrm{I}^{2}=0 \%\right)$. Better anatomical results were found for ranibizumab. Bevacizumab was associated with a $34 \%$ increase in the number of patients with at least one serious systemic adverse event (OR 1.34, 1.08 to 1.66, $\left.p=0.01, \mathrm{I}^{2}=0 \%\right)$.

Conclusions The pooled evidence confirmed that, compared with ranibizumab, bevacizumab was associated with equivalent effects on visual acuity at 1 year and with a higher risk of systemic serious adverse events. The current available data do not show which types of adverse events occur more frequently. In practice, bevacizumab should be used under a riskmanagement plan until further studies have been carried out to assess accurately the increased risk of systemic adverse events.

Keywords Bevacizumab $\cdot$ Ranibizumab $\cdot$ Neovascular age-related macular degeneration $\cdot$ Meta-analysis

\section{Introduction}

Ranibizumab, an anti-vascular endothelial growth factor (antiVEGF) monoclonal antibody fragment, was developed specifically to treat age-related macular degeneration (AMD) and was approved by the Food and Drug Administration (FDA) 
and the European Agency for the Evaluation of Medicinal Products (EMEA) in 2006 and 2007, respectively. Nevertheless, bevacizumab, a full anti-VEGF antibody derived from the same parent antibody as ranibizumab, is currently widely used off-label for AMD treatment [1].

Head-to-head studies have been performed in various countries to establish the relative efficacy of bevacizumab versus ranibizumab. The largest study, CATT (Comparison of AMD Treatments Trials), demonstrated the non-inferiority of bevacizumab versus ranibizumab on visual acuity at 1 year [2]. In this study, serious systemic adverse events occurred more frequently in the bevacizumab group, but this was not observed in the other randomised trials [3-5].

In 2012, two meta-analyses including studies with a follow-up period of 1 year were published [3, 6]. Efficacy was studied in only one of them. Chakravarthy et al. confirmed the functional equivalence of the two drugs (visual acuity) [3]. Retinal thickness at fovea was the only anatomical efficacy criterion analysed, with more favourable results for ranibizumab. The safety profiles for intravitreal injections of bevacizumab and ranibizumab appeared to be similar. Although slightly more systemic serious adverse events were observed with bevacizumab, there were no differences between the two drugs for arterial thromboembolic events, non-ocular haemorrhage or death $[3,6]$.

Since the publication of the last meta-analysis, two further head-to-head trials have been completed [5, 4]. We, therefore, decided to carry out a systematic review and meta-analysis in order to update the results on the functional and anatomical efficacy and safety profile at 1 year of bevacizumab compared with ranibizumab in patients with neovascular AMD.

\section{Methods}

The Cochrane Collaboration methods were used to perform the systematic review [7]. The meta-analysis was performed according to a protocol established before the start of the literature search and data analysis. The study was conducted and reported according to the preferred reporting items for systematic reviews and meta-analyses (PRISMA) checklist [8].

Eligibility criteria for considering studies for this review

To be eligible for inclusion, trials had to (1) compare the efficacy and/or the safety of bevacizumab and ranibizumab at 1 year (whatever the injection regimen); (2) include only patients with AMD.

Search methods for identifying studies

The systematic search was performed on Medline (from inception to October 2013), Embase (from January 2000 to
October 2013), and the Cochrane Central Register of Controlled Trials (October 2013) using relevant text words and medical subject headings that included all spellings of "macular degeneration" and "bevacizumab" (Box).

\section{Box: Trial search query}

(bevacizumab[Substance Name] OR bevacizumab[TIAB] OR Avastin[TIAB])

\section{AND}

(ranibizumab[Substance Name] OR ranibizumab[TIAB] OR Lucentis[TIAB])

\section{AND}

(Macular Degeneration[MeSH Terms] OR Macular Degeneration[TIAB] OR Macular Degenerations[TIAB] OR Age-Related Maculopathies[TIAB] OR Age Related Maculopathies[TIAB] OR Macular Dystrophy[TIAB] OR Macular Dystrophies[TIAB] OR Age-Related Macular Degeneration[TIAB] OR Age Related Macular Degeneration[TIAB] OR Age-Related Macular Degenerations[TIAB] OR Age-Related Maculopathy[TIAB] OR Age Related Maculopathy[TIAB])

\section{AND}

("randomized controlled trial" [PT] OR "controlled clinical trial[PT]" OR "randomized controlled trials"[MeSH Terms] OR "random allocation"[MeSH Terms] OR "double blind method"[MeSH Terms] OR "single-blind method"[MeSH Terms] NOT (animal NOT human)[MeSH Terms] OR "clinical trial" $[\mathrm{PT}] \mathrm{OR}$ "clin* trial*"[TIAB] OR "placebos"[MeSH Terms] OR "placebo*”[TIAB] OR "random*”[TIAB])

The search was limited to randomised clinical trials. No language restriction was applied. We checked the reference lists of the reviewed articles and original studies identified by the electronic search for other potentially eligible articles.

Trial registers were also checked for unpublished studies.

\section{Study selection}

One reviewer searched the literature and assessed the quality of trials using a standardized approach and a pre-specified protocol.

Data collection and risk of bias assessment

One reviewer extracted data from the selected trials, and two reviewers (ED, LH) checked these data for accuracy. For each trial, a standard data extraction method was used to record data on the participants' characteristics; treatment regimens; number of participants by group; primary outcome, defined as change in best corrected visual acuity (BCVA) at 1 year; anatomical efficacy parameters at baseline and achieved at the end of the follow-up (i.e., retinal thickness at fovea, intraretinal or subretinal fluid on OCT, and dye leakage on angiogram); and nature and number of serious adverse events. 
The criteria used for quality assessment were sequence generation of allocation; allocation concealment; masking of participants, staff, and outcome assessors; and other sources of bias, as recommended by the Cochrane Collaboration [7]. Studies with high or unclear risk of bias for any of the first three components were classified as low quality.

Data synthesis and analysis

The results were pooled using a random-effect model, using the odds ratio (OR) to summarize dichotomous results and the weighted mean difference (MD) to summarize continuous results, along with their $95 \%$ confidence intervals $(\mathrm{CI})$.

Statistical heterogeneity was quantified using the $\mathrm{I}^{2}$ statistic [9], which approximates the percentage of the total variation (within and between studies) that is due to between-study variation. In the absence of heterogeneity, fixed and randomeffects models yield the same results.

Potential for publication bias was explored by visually inspecting a funnel plot of the treatment effect versus standard error and Egger's test.

When needed, sensitivity analysis could be performed to determine whether some decisions had a major effect on the results of the review.

The meta-analyses were performed using R 2.15.1 (http:// www.r-project.org) with the metafor package.

\section{Results}

Characteristics of included studies

\section{Selection of studies}

Of the 51 citations retrieved from the literature search and the trial registers search, 10 studies were eligible. Three were excluded as the results were not available: the Bevacizumab Versus Ranibizumab in Age Related Macular Degeneration AxL-2009 trial (NCT01014468, status unknown); the Prevention of Vision loss in Patients with AMD by Intravitreal Injection of Bevacizumab and Ranibizumab (VIBERA, NCT00559715, status unknown); and the Lucentis Compared to Avastin Study (LUCAS, NCT01127360, completed). For one study, results were presented at a meeting, but no publications were retrieved (the Comparison of Bevacizumab and Ranibizumab in Exudative Age-Related Macular Degeneration study, BRAMD, NTR1704; 13th EURETINA Congress, 26-29 September 2013, Hamburg).

One trial was excluded $[10,11]$ due to the availability of two papers for this same trial with large discrepancies between the two papers regarding data and results (notably the sample size), which meant it was impossible to choose between these two different versions.

Finally, five studies were included (Fig. 1): Subramanian et al. [12]; the CATT study [2]; the Alternative Treatments to Inhibit VEGF in Age-Related Choroidal Neovascularization (IVAN) study [3]; the Multicentre Anti-VEGF Trial in Austria (MANTA) [5]; and the French study group Avastin versus Lucentis for Neovascular AMD (GEFAL) [4].

\section{Methodology of the studies}

Overall, two treatment regimens were identified: monthly intravitreal injections, and as-needed (Table 1). CATT presented separate results of bevacizumab versus ranibizumab (i) with monthly intravitreal injections and (ii) with an asneeded regimen; therefore, it was considered as two sets of data. Finally, six sets were included in the quantitative analysis.

The primary outcome for all trials was defined as change in best corrected visual acuity (BCVA) at 1 year. Three trials were primarily designed as non-inferiority trials (CATT, IVAN, and GEFAL); the non-inferiority margins were -5 , -3.5 , and -5 , respectively. The two others were considered as superiority trials (expected difference between treatments of seven letters for MANTA and no hypothesis defined in Subramanian et al.).

In the GEFAL and MANTA studies, participants, investigators, and outcome assessors were masked. One trial was described as single-masked (CATT), but for the betweentreatments comparison, the investigator and assessor were masked. For the IVAN trial, $98.6 \%$ of the participants and $98.7 \%$ ophthalmologists were masked at the 12 -month visit. The Subramanian et al. study was described as doublemasked, with no further details reported.

Three trials (CATT, IVAN, and GEFAL) have reported adverse events according to the Medical Dictionary for Regulatory Activities (MedDRA) system.

Selective outcome bias was low in all five trials regarding visual acuity endpoints and serious adverse events (only one trial did not provide adverse events in detail [12]).

Data analysis and synthesis

\section{Patient characteristics}

Overall, 2,686 patients were randomised to one of the two drugs (Table 1). The baseline characteristics of patients are presented in Table 2.

\section{Functional endpoint (visual acuity)}

The analysis population for the primary outcome consisted of 993 patients for bevacizumab and 1,028 for ranibizumab 


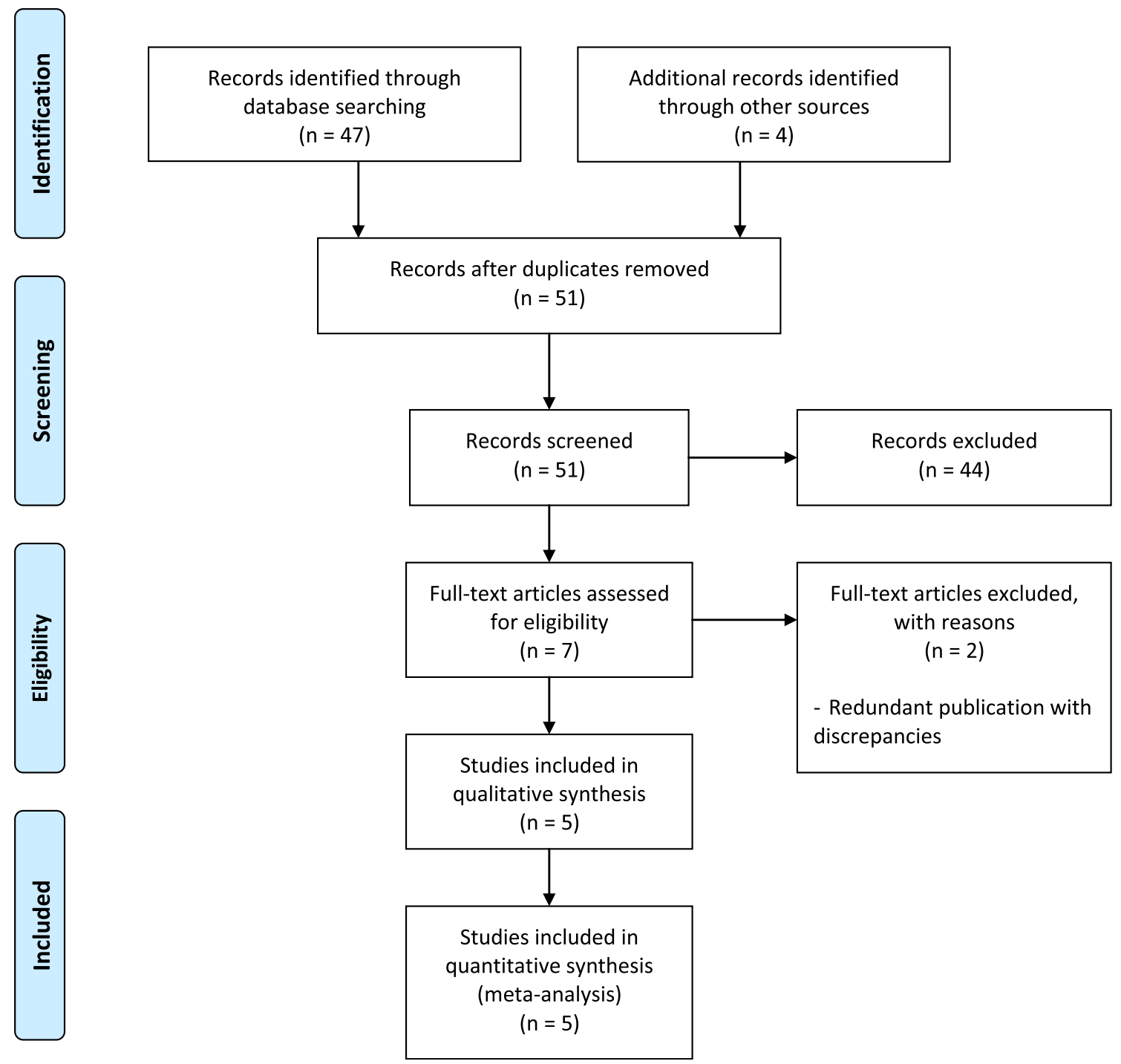

Fig. 1 Study identification and selection flowchart

Table 1 Characteristics of the studies included in the quantitative meta-analysis

\begin{tabular}{|c|c|c|c|c|c|c|c|}
\hline Study & Setting & Location & $\begin{array}{l}\text { Treatment } \\
\text { regimen }\end{array}$ & $\begin{array}{l}\text { Number of patients } \\
\text { in the considered } \\
\text { population for safety } \\
\text { (bevacizumab/ } \\
\text { ranibizumab) }\end{array}$ & $\begin{array}{l}\text { Analysis } \\
\text { Population } \\
\text { for primary } \\
\text { outcome }\end{array}$ & $\begin{array}{l}\text { Number of } \\
\text { patients in the } \\
\text { considered } \\
\text { population for } \\
\text { primary outcome } \\
\text { (bevacizumab/ } \\
\text { ranibizumab) }\end{array}$ & $\begin{array}{l}\text { Number of } \\
\text { randomised } \\
\text { patients } \\
\text { (bevacizumab/ } \\
\text { ranibizumab) }\end{array}$ \\
\hline GEFAL, $2013^{4}$ & Multi-centre & France & As-needed & $255 / 246$ & Per protocol & $191 / 183$ & $246 / 239$ \\
\hline MANTA, $2013^{5}$ & Multi-centre & Austria & As-needed & 321 overall & ITT & $154 / 163 *$ & $154 / 163$ \\
\hline IVAN, $2012^{3}$ & Multi-centre & England & $\begin{array}{l}\text { As-needed and } \\
\text { Monthly }\end{array}$ & $305 / 323$ & ITT & $251 / 269$ & $296 / 314$ \\
\hline CATT (monthly), $2011^{2}$ & Multi-centre & USA & Monthly & 1,208 overall & ITT & $265 / 284$ & $286 / 301$ \\
\hline CATT (as needed), $2011^{2}$ & Multi-centre & USA & As-needed & & ITT & $271 / 285$ & $300 / 298$ \\
\hline Subramanian et al., $2010^{12}$ & Single centre & USA & As-needed & $20 / 8$ & Available patients & $15 / 7$ & $20 / 8$ \\
\hline
\end{tabular}

ITT intention to treat

${ }^{\text {a }}$ Specific data were not available to be included in the meta-analysis 


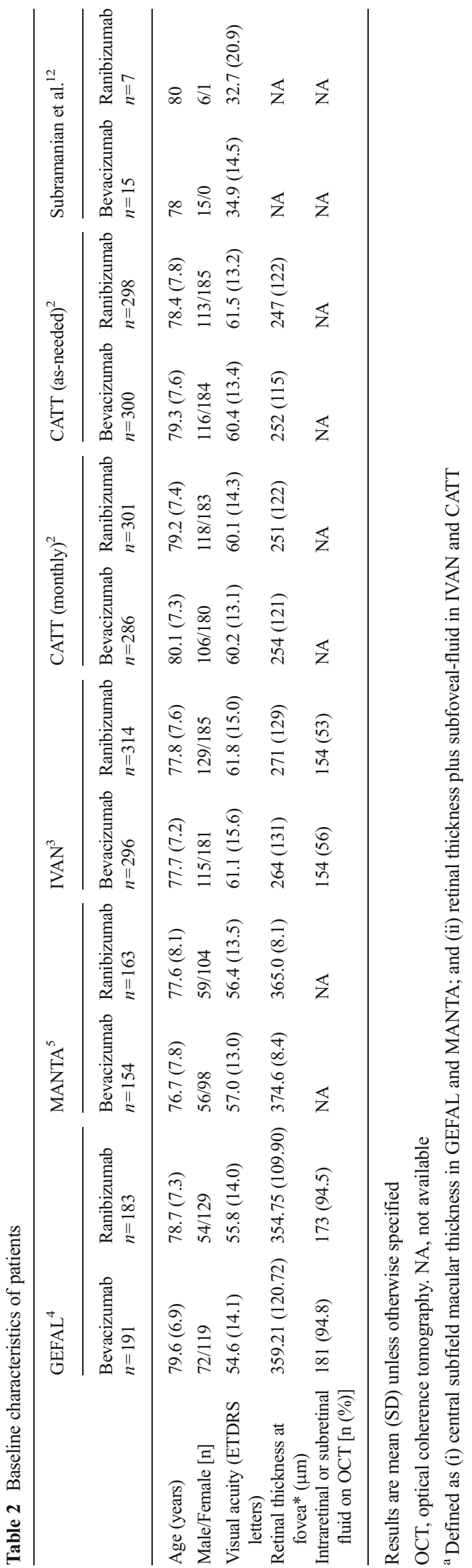

(MANTA did not provide the mean change in BCVA in each group and was, therefore, not included). The difference between bevacizumab and ranibizumab in terms of mean change in BCVA at 1 year was not significant (MD -0.57 letters, -1.80 to $0.66, p=0.37, \mathrm{I}^{2}=0 \%$; Fig. 2 ). The difference was not significant for any other visual acuity parameters.

\section{Anatomical endpoints}

Overall, ranibizumab was associated with better anatomical outcomes (Fig. 3) in terms of change in retinal thickness at fovea at 12 months (MD 13.77, 2.05 to $25.48, p=0.02$ ), the presence of intraretinal or subretinal fluid on OCT (OR 1.56, 1.29 to $1.89, p<0.01)$ and dye leakage on angiogram (OR $1.23,1.02$ to $1.49, p=0.03$ ).

\section{Adverse events}

The adverse events are presented in Table 3.

Systemic (i.e. excluding ocular events) adverse event rates were available for all the five trials. Bevacizumab was associated with a $34 \%$ increase in the risk of experiencing at least one serious systemic adverse event (OR 1.34, 1.08 to $1.66, p=$ 0.01 ; Fig. 4) compared with ranibizumab, with no heterogeneity detected $\left(\mathrm{I}^{2}=0 \%\right)$. No evidence of publication bias was suggested for this endpoint by visual inspection of funnel plots or by Egger's test (two-tailed p-value of 0.17). Among the serious systemic adverse events taken individually, none were both consistently and significantly increased (Table 3 ). Infection was associated with a non-significant increase that was relatively consistent across the trials. An increase in gastrointestinal disorders was found with the fixed effect model, but not with the random effect model, perhaps due to heterogeneity.

A total of 33 ocular serious adverse events were reported in the trials. They were not found to be different between bevacizumab and ranibizumab (Table 3). Endophthalmitis were reported in two trials (CATT, GEFAL) and bevacizumab was not associated with a significant difference. Only one trial reported a case of uveitis (IVAN).

\section{Sensitivity analysis}

We tested the robustness of our analyses by performing sensitivity analyses excluding the CATT study (largest trial). When excluding data from the two sets of CATT, the systemic adverse effects were not statistically different between the groups (OR 1.33, 0.95 to $1.86, p=0.09$ ). We also performed analyses excluding alternatively CATT monthly, CATT as needed, GEFAL, and IVAN, thus ensuring similar sample size in each comparison. Only analyses including one of the CATT dataset were found significant. 


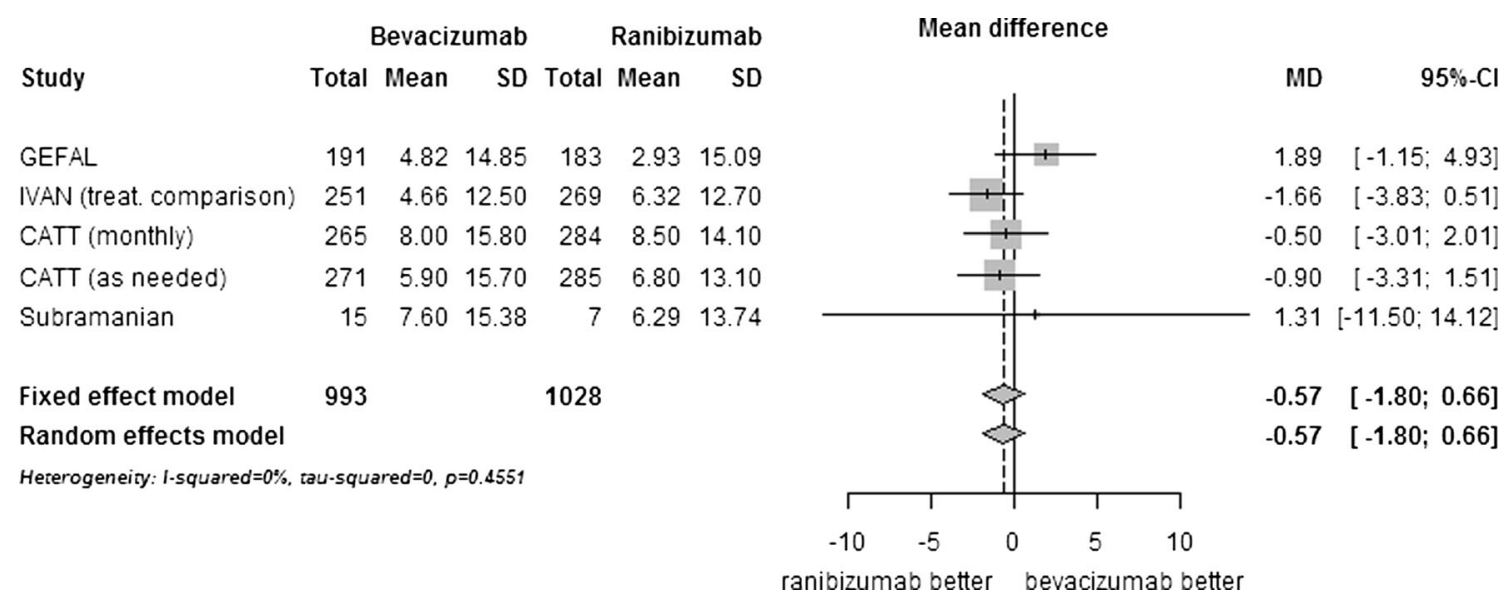

Fig. 2 Comparison of bevacizumab with ranibizumab for mean change in visual acuity (ETDRS letters) at 1 year. CI, confidence interval; MD, mean difference; $\mathrm{SD}$, standard deviation

\section{Discussion}

This meta-analysis, updated with the inclusion of 2013 data from the MANTA and the GEFAL studies, did not show any difference between bevacizumab and ranibizumab in terms of the change in BCVA at 1 year in neovascular AMD.

The mean difference was -0.57 letters with a lower limit in the $95 \%$ confidence interval of -1.80 letters. This lower bound is above all the non-inferiority margins chosen in the non-inferiority trials $(-3.5$ to -5$)$. These results complete those of the previous meta-analysis and support the functional non-inferiority of bevacizumab over ranibizumab.

This meta-analysis also confirmed the trend observed in all head-to-head studies: better anatomical results for ranibizumab, with decrease in retinal thickness at fovea, fluid, and dye leakage at 1 year. Results for retinal thickness at fovea should be considered with caution since the definitions varied between studies. The results for fluid and dye leakage have never previously been studied in previous meta-analyses. Further studies with longer follow-up periods would be required to confirm if the improvement in anatomical outcomes observed with ranibizumab is maintained over time, thus impacting on visual function and quality of life.

Concerning safety, the combined results showed a significant increase in systemic serious adverse events of more than $30 \%$ with bevacizumab compared with ranibizumab. The two previous meta-analyses had already reported this increase, with relative risks for systemic adverse events of $1.35(95 \%$ CI 1.05 to $1.72,1,795$ participants) [3] and 1.3 (95\% CI 1.0 to 1.7, cumulative data from CATT publication) [6] at 1 year.

The excess risk of experiencing at least one systemic adverse event observed with bevacizumab could be surprising for a drug administered locally. Three studies demonstrated that the VEGF plasma level was significantly lower with intravitreal bevacizumab than with intravitreal ranibizumab $[3,13,14]$. VEGF is fundamental for numerous physiological extraocular functions, but the link between a lower VEGF serum concentration and higher serious adverse events has not yet been proven. Understanding the mechanism by which the systemic diffusion and action of bevacizumab occur after intravitreal injections, and identifying the potential risk factors associated with this mechanism, could contribute to identifying which patients should be treated with caution when undergoing bevacizumab intravitreal treatment.

However, serious systemic adverse events would require more advanced analysis. Indeed, our sensitivity analyses tended to lower the effect. Although the treatment effect size was similar across studies, the rate of events differed between CATT and other studies ( $20 \%$ of events versus $11 \%$ ), thus suggesting that there might be some heterogeneity.

None of the side effects of bevacizumab seen after systemic use in the early wet AMD studies [15], especially hypertension, or the cancer studies could be confirmed in any of the head-to-head wet AMD-studies. However, none of the five studies included in this meta-analysis was designed or powered to assess safety concerns.

Moreover, as no serious adverse event taken individually was significantly increased in our analysis that included 2,625 patients (taking into account the heterogeneity), it is not possible to explain clearly this overall excess of systemic adverse events. A safety signal could be suspected for infections. This signal was found consistently in all the trials included in this meta-analysis, but was not statistically significant. Given the safety profile of systemic administrations of bevacizumab, the recommendation could be to monitor patients closely for infection. Especially, cases of epidemic endophthalmitis have been previously reported, and might be increased due to repackaging of vials of bevacizumab in single syringes in the absence of a commercially specific packaging for intravitreal administration. For the preparation of off-label bevacizumab, one could recommend either the use of one vial per patient such as in GEFAL, or, from a money-saving perspective, an industrial repackaging in an aseptic filing facility such as in CATT. 


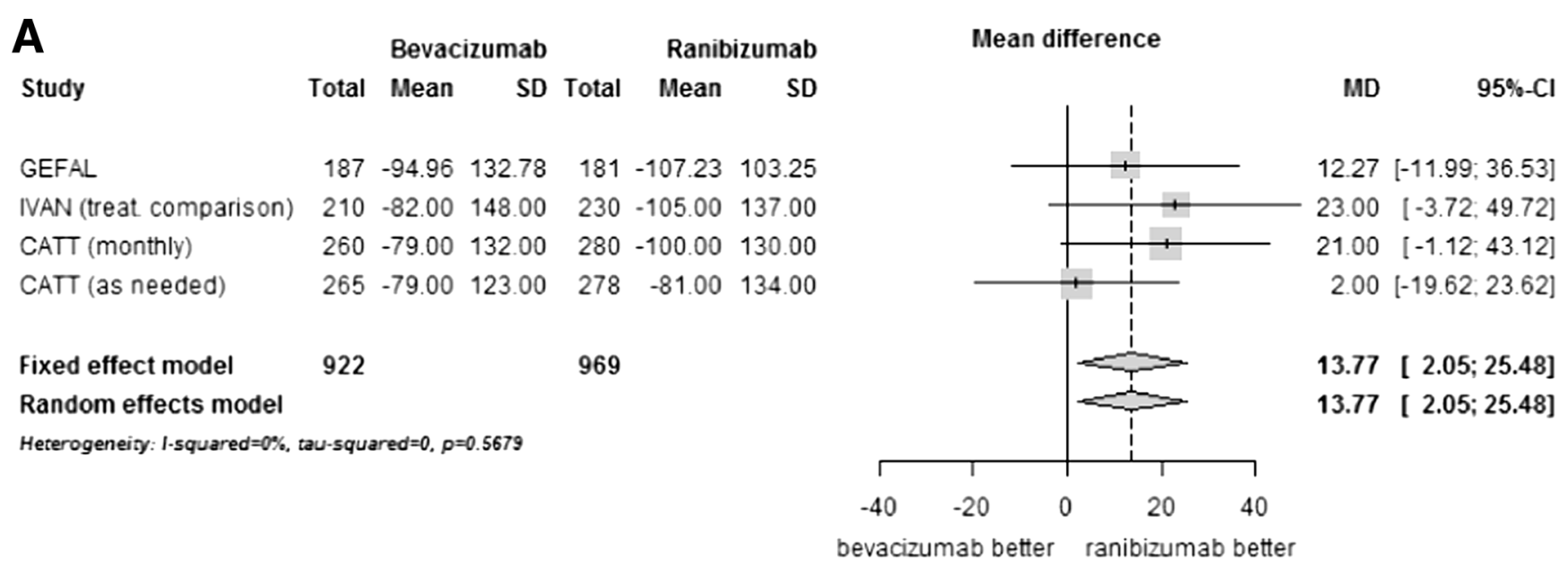

B

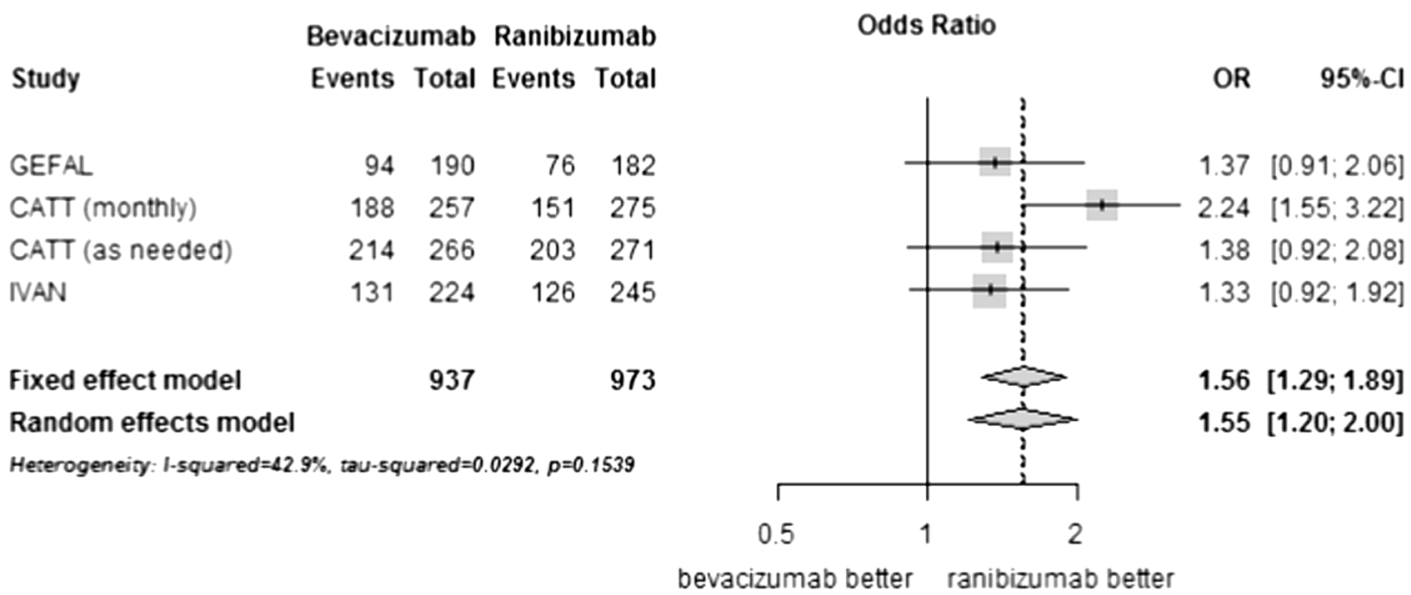

C

\begin{tabular}{lrrrr} 
Study & \multicolumn{3}{c}{$\begin{array}{l}\text { Bevacizumab Ranibizumab } \\
\text { Events Total Events }\end{array}$} \\
& 84 & 170 & 66 & 160 \\
GEFAL & 100 & 253 & 97 & 264 \\
CATT (monthly) & 145 & 256 & 137 & 270 \\
CATT (as needed) & 86 & 199 & 82 & 211 \\
IVAN & & & &
\end{tabular}

Fixed effect model

878

905

Random effects model

Heterogeneity: $1 \cdot$ squared $=0 \%$, tau.squared $=0, p=0.8972$

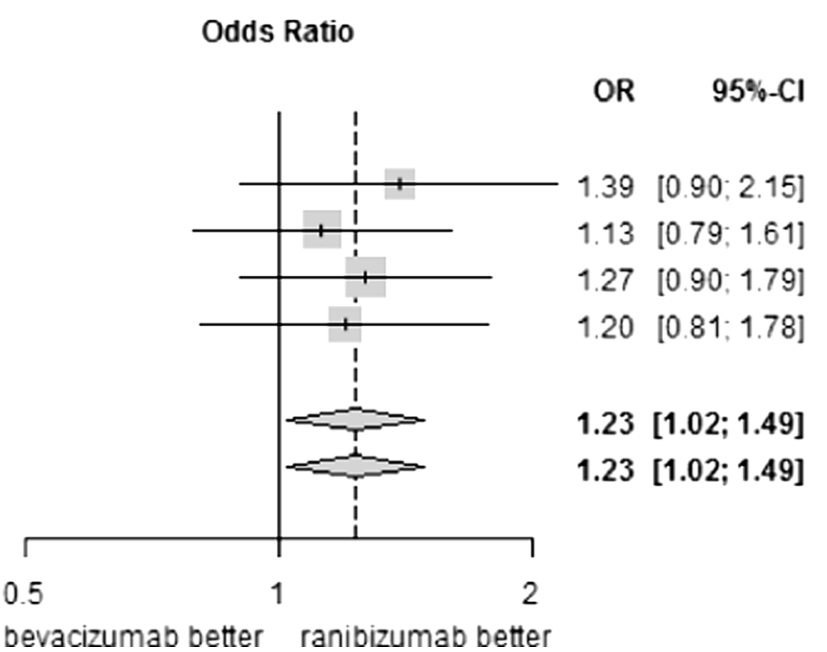

Fig. 3 Comparison of bevacizumab with ranibizumab for anatomical results. Panel a, Change in retinal thickness at fovea at 1 year (microns); Panel b, Presence of intraretinal or subretinal fluid on optical coherence

tomography at 1 year; Panel $\mathbf{c}$, Presence of dye leakage on angiogram at 1 year. CI, confidence interval; MD, mean difference; OR, Odds ratio; $\mathrm{SD}$, standard deviation

This meta-analysis is the most comprehensive review of literature assessing the relative efficacy and safety of bevacizumab and ranibizumab in neovascular AMD.
Despite the absence of statistical heterogeneity for most of the outcomes studied, the selected studies were clinically heterogeneous in terms of treatment regimens. Moreover, their 
Table 3 Safety results

\begin{tabular}{|c|c|c|c|c|c|c|}
\hline \multirow[t]{2}{*}{ Endpoints } & \multirow[t]{2}{*}{ Number of study sets } & \multirow[t]{2}{*}{$\mathrm{I}^{2} \dagger(\%)$} & \multicolumn{2}{|l|}{ Fixed effect } & \multicolumn{2}{|l|}{ Random effect } \\
\hline & & & OR $[95 \% \mathrm{CI}]$ & $\mathrm{p}$ value & OR $[95 \% \mathrm{CI}]$ & $\mathrm{p}$ value \\
\hline Systemic* serious adverse events & 6 & 0 & $1.34[1.08 ; 1.66]$ & 0.01 & $1.34[1.08 ; 1.66]$ & 0.01 \\
\hline Gastrointestinal disorder & 5 & 42 & $2.23[1.09 ; 4.57]$ & 0.03 & $2.19[0.77 ; 6.21]$ & 0.14 \\
\hline Infection & 5 & 0 & $1.61[0.99 ; 2.64]$ & 0.06 & $1.61[0.98 ; 2.64]$ & 0.06 \\
\hline Nervous system disorder & 5 & 7 & $1.07[0.61 ; 1.87]$ & 0.82 & $1.04[0.55 ; 1.95]$ & 0.91 \\
\hline Benign or malignant neoplasm & 5 & 0 & $0.97[0.52 ; 1.81]$ & 0.93 & $0.93[0.49 ; 1.77]$ & 0.83 \\
\hline Cardiac disorder & 4 & 15 & $1.06[0.66 ; 1.69]$ & 0.82 & $1.04[0.60 ; 1.78]$ & 0.89 \\
\hline Myocardial infraction & 5 & 0 & $0.83[0.32 ; 2.10]$ & 0.69 & $0.85[0.32 ; 2.24]$ & 0.74 \\
\hline Stroke & 6 & 0 & $0.67[0.23 ; 1.97]$ & 0.47 & $0.76[0.23 ; 2.46]$ & 0.65 \\
\hline APTC arterial thromboembolic events & 5 & 0 & $0.82[0.42 ; 1.59]$ & 0.55 & $0.89[0.44 ; 1.80]$ & 0.76 \\
\hline Venous thrombotic event & 4 & 0 & $2.78[0.82 ; 9.45]$ & 0.10 & $2.38[0.57 ; 9.84]$ & 0.23 \\
\hline Death from any cause & 6 & 0 & $1.32[0.74 ; 2.36]$ & 0.35 & $1.30[0.71 ; 2.36]$ & 0.39 \\
\hline Endophthalmitis & 4 & 4 & $1.33[0.33 ; 5.37]$ & 0.69 & $1.37[0.29 ; 6.53]$ & 0.69 \\
\hline Serious non-ocular haemorrhage & 2 & 0 & $3.77[0.62 ; 22.90]$ & 0.15 & $3.36[0.52 ; 21.78]$ & 0.20 \\
\hline Ocular Serious adverse events & 5 & 57 & $1.78[0.87 ; 3.63]$ & 0.11 & $1.62[0.49 ; 5.39]$ & 0.43 \\
\hline
\end{tabular}

APTC Antiplatelet trialist collaboration, $C I$ confidence interval, $O R$ odds ratio

*Excluding ocular events

$\uparrow$ Statistical heterogeneity

statistical perspectives (non-inferiority or superiority) and populations (intention-to-treat or per protocol sets) also differed. Finally, the final BCVA value used in the analyses varied, as some trials performed imputations for missing data at 1 year.

However, the objective of the meta-analysis was to analyse the effect of the treatment independently of the treatment regimen; the duration and doses of the drugs were identical for most of the studies. Furthermore, there was no random error for mean change in BCVA at 1 year (primary efficacy outcome) as no heterogeneity was found between the results of the trials.

The analysis of safety events is also prone to several biases as the data varied in each study in terms of quality, incidence, severity, and adjudication. In RCTs, all SAEs must be specifically investigated and recorded regardless of the imputability with the drug. The reporting may also be influenced by the expectations of the investigators, sponsors, and patients. However, the definitions for safety outcomes were based on the MedDRA system for three out of five selected trials, representing 2,280 of

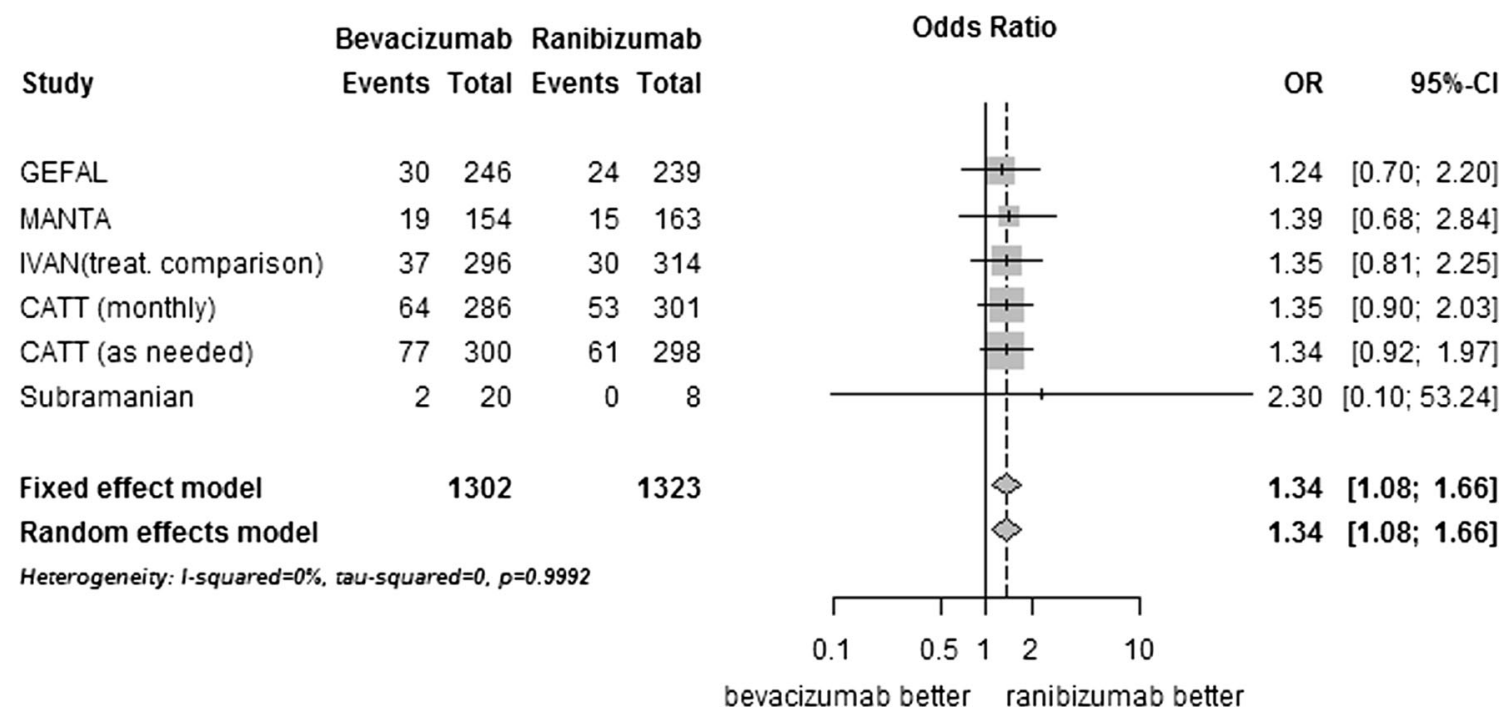

Fig. 4 Comparison of bevacizumab with ranibizumab for systemic serious adverse events at 1 year. CI, confidence interval; MD, mean difference; OR, odds ratio; $\mathrm{SD}$, standard deviation 
the 2,625 patients in the safety population. Despite the exploratory nature of the safety analysis, no heterogeneity was observed, which reinforces our results on systemic serious adverse events as it means they cannot be imputed to artifactual data.

A further limitation to this review is that at least four more trials comparing bevacizumab and ranibizumab for visual acuity (primary outcome measured at 1 year) in AMD have been identified, but at the time of our search, the results were not published.

This meta-analysis found sufficient evidence to conclude that bevacizumab is associated with similar effects on visual acuity compared with ranibizumab. It also showed that bevacizumab may be associated with an excessive risk of systemic serious adverse events. However, the current available data do not show which types of adverse events occur more frequently. In practice, bevacizumab for neovascular AMD should be used under a risk management plan. The main explanation for the current use of bevacizumab is economic, reinforced by an equivalent functional efficiency with ranibizumab, but this should be balanced against the poorer anatomical results and a suspected higher rate of serious systemic adverse events than ranibizumab at 1 year.

Acknowledgments We thank Michel Cucherat for his methodological advice and his help in designing the meta-analysis.

\section{Funding source None}

Meeting Presentation Some of the results of this analysis were presented at the ARVO meeting (Seattle, WA, USA) on May 7, 2013, the SFO meeting (Paris, France) on May 11, 2013, and the EURETINA congress (Hamburg, Germany) on October 26, 2013.

Conflict of interest - LK has been principal investigator for trials sponsored by Novartis, Bausch\&Lomb, Théa, and Alcon; has sat on advisory boards for Alcon, Alimera Sciences, Allergan, Bayer, Bausch\&Lomb, Novartis, and Théa; and has received lecture fees from Alcon, Allergan, Bayer, Bausch\&Lomb, Krys group, Novartis, Théa, and Zeiss.

- EHS has received honoraria from Novartis, Bayer, and Allergan; has sat on advisory boards for Bausch \& Lomb and Théa; has received lecture fees from Bausch\&Lomb; and has received consulting fees from Novartis, Bayer, and Allergan.

- JFG has sat on advisory boards for Novartis; has received lecture fees from Allergan and Bayer; and has received consulting fees from Allergan, Bayer, and Sanofi-Fovea

- FC, ED, EED, and LH declared no conflict of interests.

Open Access This article is distributed under the terms of the Creative Commons Attribution License which permits any use, distribution, and reproduction in any medium, provided the original author(s) and the source are credited.

\section{References}

1. Stein JD, Hanrahan BW, Comer GM, Sloan FA (2013) Diffusion of technologies for the care of older adults with exudative age-related macular degeneration. Am J Ophthalmol 155(4):688-696. doi:10. 1016/j.ajo.2012.10.003, 696 e681-682
2. Martin DF, Maguire MG, Ying GS, Grunwald JE, Fine SL, Jaffe GJ (2011) Ranibizumab and bevacizumab for neovascular age-related macular degeneration. N Engl J Med 364(20):1897-1908. doi:10. 1056/NEJMoa1102673

3. Chakravarthy U, Harding SP, Rogers CA, Downes SM, Lotery AJ, Wordsworth S, Reeves BC (2012) Ranibizumab versus bevacizumab to treat neovascular age-related macular degeneration: one-year findings from the IVAN randomized trial. Ophthalmology 119(7):13991411. doi:10.1016/j.ophtha.2012.04.015

4. Kodjikian L, Souied EH, Mimoun G, Mauget-Faysse M, BeharCohen F, Decullier E, Huot L, Aulagner G (2013) Ranibizumab versus Bevacizumab for neovascular age-related macular degeneration: results from the GEFAL Noninferiority randomized trial. Ophthalmology 120(11):2300-2309. doi:10.1016/j.ophtha.2013. 06.020

5. Krebs I, Schmetterer L, Boltz A, Told R, Vecsei-Marlovits V, Egger S, Schonherr U, Haas A, Ansari-Shahrezaei S, Binder S (2013) A randomised double-masked trial comparing the visual outcome after treatment with ranibizumab or bevacizumab in patients with neovascular age-related macular degeneration. Br J Ophthalmol 97(3):266-271. doi:10.1136/bjophthalmol-2012-302391

6. Schmucker C, Ehlken C, Agostini HT, Antes G, Ruecker G, Lelgemann M, Loke YK (2012) A safety review and meta-analyses of bevacizumab and ranibizumab: off-label versus goldstandard. PLoS One 7(8):e42701. doi:10.1371/journal.pone.0042701

7. Cochrane Handbook for Systematic Reviews of Interventions, Version 5.1.0 [updated March 2011]. The Cochrane Collaboration, 2011. Available from http://handbook.cochrane.org/

8. Moher D, Liberati A, Tetzlaff J, Altman DG (2009) Preferred reporting items for systematic reviews and meta-analyses: the PRISMA statement. BMJ 339:b2535. doi:10.1136/bmj.b2535

9. Higgins JP, Thompson SG, Deeks JJ, Altman DG (2003) Measuring inconsistency in meta-analyses. BMJ 327(7414):557-560. doi:10. 1136/bmj.327.7414.557

10. Biswas P, Sengupta S, Choudhary R, Home S, Paul A, Sinha S (2011) Comparative role of intravitreal ranibizumab versus bevacizumab in choroidal neovascular membrane in age-related macular degeneration. Indian J Ophthalmol 59(3):191-196. doi:10.4103/0301-4738. 81023

11. Biswas P, Sengupta S, Choudhary R, Home S, Paul A, Sinha S (2011) Comparing ranibizumab with bevacizumab. Ophthalmology 118(3): 600-600. doi:10.1016/j.ophtha.2010.10.027, e602

12. Subramanian ML, Abedi G, Ness S, Ahmed E, Fenberg M, Daly MK, Houranieh A, Feinberg EB (2010) Bevacizumab vs ranibizumab for age-related macular degeneration: 1-year outcomes of a prospective, double-masked randomised clinical trial. Eye (Lond) 24(11):1708-1715. doi:10.1038/eye. 2010.147

13. Carneiro AM, Costa R, Falcao MS, Barthelmes D, Mendonca LS, Fonseca SL, Goncalves R, Goncalves C, Falcao-Reis FM, Soares R (2012) Vascular endothelial growth factor plasma levels before and after treatment of neovascular age-related macular degeneration with bevacizumab or ranibizumab. Acta Ophthalmol 90(1):e25-e30. doi: 10.1111/j.1755-3768.2011.02240.x

14. Zehetner C, Kirchmair R, Huber S, Kralinger MT, Kieselbach GF (2013) Plasma levels of vascular endothelial growth factor before and after intravitreal injection of bevacizumab, ranibizumab and pegaptanib in patients with age-related macular degeneration, and in patients with diabetic macular oedema. Br J Ophthalmol 97(4): 454-459. doi:10.1136/bjophthalmol-2012-302451

15. Moshfeghi AA, Rosenfeld PJ, Puliafito CA, Michels S, Marcus EN, Lenchus JD, Venkatraman AS (2006) Systemic bevacizumab (Avastin) therapy for neovascular age-related macular degeneration: twenty-four-week results of an uncontrolled open-label clinical study. Ophthalmology 113(11):2002.e2001-2012. doi:10.1016/j.ophtha. 2006.05.070 